\title{
Pronóstico de la ocurrencia de precipitación diaria en un gradiente altitudinal en el Sur del Ecuador, mediante un generador climático
}

\section{(Forecasting of daily precipitation occurrence in an altitudinal gradient in southern Ecuador using a weather generator)}

\author{
Diego Urdiales ${ }^{1}$, Rolando Célleri ${ }^{1}$
}

\begin{abstract}
Resumen:
El pronóstico de precipitación es fundamental para mejorar la gestión de los recursos hídricos tanto para proyectos de aprovechamiento como de reducción de riesgos. Debido a su alta variabilidad, es un reto realizar un buen pronóstico de lluvia. En el presente estudio se empleó un generador climático (GC) para investigar la calidad del pronóstico de ocurrencia de precipitación diaria en una zona de montaña. El GC implementado usó como predictores a tres variables: la variable binaria ocurrencia del día anterior $\left(\mathrm{K}_{\mathrm{t}-1}\right)$, las temperaturas máximas y mínimas del día anterior ( $\mathrm{Tx}_{\mathrm{t}-1}, \mathrm{Tn}_{\mathrm{t}-1}$, respectivamente); y dos covariables: las anomalías de la Temperatura mensual Superficial del Mar de las Regiones 1+2 y 3.4 de El Niño-Oscilación del Sur (Niño 1+2 y Niño 3.4 respectivamente). Determinamos que las variables $\mathrm{K}_{\mathrm{t}-1}, \mathrm{Tn}_{\mathrm{t}-1}$ y la covariable Niño 3,4 mejoran el ajuste en el pronóstico. Encontramos diferencias notables en el número de días consecutivos secos y lluviosos en el gradiente altitudinal en un período lluvioso. El estudio permitió el planteamiento de varias hipótesis a partir del uso del GC las cuales ayudan a la comprensión del funcionamiento del sistema climático y el mejoramiento del pronóstico de precipitación.
\end{abstract}

Palabras clave: Pronóstico; Niño 3.4; generador climático; gradiente altitudinal, páramo.

\begin{abstract}
:
Precipitation forecast is fundamental for improving the management of water resources, for development projects and risk reduction. Due to its high variability, high quality rain forecasts are still a challenge. In the present study, a weather generator (WG) was used to study the quality of the forecast of daily rainfall occurrence. The WG was implemented using three variables as predictors: the binary variable precipitation occurrence of the previous day $\left(\mathrm{K}_{\mathrm{t}-1}\right)$, the maximum and minimum temperatures of the previous day ( $\mathrm{Tx}_{\mathrm{t}}$ 1, $\mathrm{Tn}_{\mathrm{t}-1}$, respectively); and two co-variables: monthly values of the observed Sea Surface Temperature Anomalies of the Regions $1+2$ and 3.4 of El Niño-Southern Oscillation (Niño $1+2$ and Niño 3.4 respectively). We found that the variables $\mathrm{K}_{\mathrm{t}-1}, \mathrm{Tn}_{\mathrm{t}-1}$ and $\mathrm{co}^{-}$ variable Niño 3.4 are those that improve the performance of the precipitation occurrence forecasting. There was a noticeable difference in the number of consecutive wet and dry spell days in the altitudinal gradient in a rainy period. We propose several hypotheses based on the use of WG, which allow the understanding of the functioning of the climate system and the improvement of the forecast of precipitation occurrence in a mountainous area.
\end{abstract}

Keywords: Forecasting, Niño 3.4, weather generator, altitudinal gradient, páramo.

\footnotetext{
${ }_{1}$ Departamento de Recursos Hídricos y Ciencias Ambientales. Universidad de Cuenca, Cuenca, Ecuador (dhurdiales@uc.cl; rolando.celleri@ucuenca.edu.ec).
} 


\section{Introducción}

La precipitación es considerada como el componente básico del ciclo hidrológico global y es responsable de la deposición de agua dulce en la Tierra. El agua es utilizada en usos consuntivos y no consuntivos tanto para la sociedad humana y los ecosistemas naturales (González et al., 2015), por lo que su monitoreo, análisis y pronóstico a diferentes escalas espacio-temporales juega un rol importante para la toma de medidas de adaptación a eventos hidrometeorológicos extremos y al cambio climático (Shi et al., 2016).

El clima del Ecuador se zonifica en tres regiones climáticas principales (Ballari et al., 2018), las cuales se modifican debido a la Zona de Convergencia Intertropical (ZCIT), las Perturbaciones de la Amazonía, la Vaguada del Perú, el Alta Semipermanente del Pacífico Suroriental y de la conocida Alta del Caribe, EI Niño Oscilación del Sur (ENOS) es una de las fuentes predominante de variabilidad interanual de lluvia (Francou et al., 2004; Ropelewski et al., 1996; Vuille et al., 2005; Walker, 1924), produce una precipitación superior/inferior a la normal durante los períodos de El Niño/La Niña. Las llanuras costeras del Pacífico presentan una estación lluviosa de diciembre a abril. El clima en esta región está fuertemente influenciado por la temperatura de la superficie del mar del Océano Pacífico; sin embargo, no hay un consenso científico respecto a la influencia de los diferentes índices ENOS en zonas andinas del país. Por ejemplo, Rossel et al., (1999) argumentan que la influencia de EI Niño 1+2 a una escala anual afecta regiones por debajo de 1200 m sobre el nivel del mar (m s.n.m), conocidas como llanuras costeras. De igual forma, Campozano et al., (2014) concluyen que la mayor parte del país es afectada por El Niño 1+2. Asimismo, (Vicente-Serrano et al., 2017) asume que la variabilidad interanual en zonas altoandinas está influenciada por el Niño 3.4 y en planicies costeras por El Niño 1+2. A escalas intranuales, el anticiclón del sudeste del Pacífico inhibe la precipitación durante octubre y noviembre en esta región. Las montañas de Los Andes muestran dos estaciones lluviosas, de febrero a mayo y de octubre a noviembre, impulsadas principalmente por la ZCIT (Celleri et al., 2007). En la selva amazónica, se agrega un tercer pico a la señal bimodal durante julio; por lo tanto, la lluvia en esta región está constantemente presente durante todo el año. El efecto de ENOS en el Amazonas es opuesto a las llanuras costeras; sin embargo, durante los períodos muy fuertes de El Niño, se observa una precipitación superior a la media anual normal (Ulloa et al., 2017).

Los pronósticos climáticos numéricos (PCN) respaldan la gestión de emergencias, mitigación de impactos y prevención de pérdidas económicas producidas por eventos extremos (sequías e inundaciones). Por ejemplo, los sistemas de riego son deficientes en temporadas secas y comúnmente la irrigación se realiza por la noche (De Biévre et al., 2003), entonces un PCN será capaz de cuantificar el ingreso de precipitación al sistema y así abarcar la demanda de los usuarios de riego. En los últimos años, los mayores esfuerzos en las predicciones (pronósticos), proyecciones y simulaciones se han centrado en el componente de precipitación ya que si existe una deficiente representación de la lluvia habrá una exigua caracterización de otras variables meteorológicas (Srikanthan et al., 2001; Wan et al., 2005). La variabilidad de la precipitación se debe a la topografía (orientación, elevación y pendiente de las montañas) e influencias climáticas (viento predominante, distancia al mar) alteradas por el aire húmedo que bloquea las montañas (Kumar, 2015; Wastl et al., 2010). De hecho, existen varios estudios al norte de la cordillera de Los Andes, que demuestran una notable variabilidad espacio-temporal (Buytaert et al., 2006; Celleri et al., 2007; Espinoza et al., 2009). Por ejemplo, las precipitaciones anuales en el páramo andino varían desde $700 \mathrm{~mm}$ hasta $3000 \mathrm{~mm}$ (Rollenbeck et al., 2011), mientras que los valores para los páramos ecuatorianos y peruanos se encuentran entre 1010 y 1390 mm (Ochoa et al., 2016; Rollenbeck et al., 2011). Adicionalmente, Muñoz et al., (2016) indican que incluso a nivel local existe una prominente variabilidad de la precipitación, lo cual se demuestra en su estudio a lo largo de un gradiente altitudinal 2500 - 3900 m s.n.m., lo cual promueve que el pronóstico de lluvia en una cuenca altoandina sea complejo y un reto, pues la mayoría de estudios de pronóstico de precipitación se han 
realizado con una sola estación pluviométrica debido a una deficiente distribución espacial de estaciones (p. ej. Avilés et al., (2015)). Por lo tanto, un modelo calibrado en un solo sitio no será generalizable para las condiciones variables de Los Andes, aun para cuencas de mesoescala (menos de $1000 \mathrm{~km}^{2}$ ).

Los avances en PCN representan una revolución silenciosa. Hasta la actualidad, en zonas tropicales no se han llegado a pronósticos efectivos (sobre el 50\%) en escala de diez días (Bauer et al., 2015). Las tres etapas de PCN son: a) Representación de procesos físicos, b) Ensamble de pronósticos y c) Iniciación de un modelo (Kalnay, 2003). En zonas montañosas, por ejemplo en Los Alpes suizos, el mejoramiento de los pronósticos de precipitación se ha enfocado en las observaciones sinópticas, conjuntamente con la ayuda de radares meteorológicos de largo alcance y una red densa de datos históricos (Cherubini et al., 2002; Sokol et al., 2009). En América del Sur, Chou et al., (2005) han obtenido pronósticos de precipitación robustos a escala mensual mediante el modelo determinístico Eta. Asimismo, los Modelos Estocásticos o Generadores Climáticos (GCs) han sido ampliamente implementados para la cuantificación y comportamiento de procesos meteorológicos (p. ej. Mishra et al., (2005)para la detección de sequías, para el análisis de probabilidades de ocurrencia de precipitación Caloiero et al., (2016) y para el pronóstico mensual de sequías Avilés et al., (2015).

Los GCs pueden representar efectivamente la variabilidad intrínseca natural (estocástico) de los procesos de precipitación, con prominente enfoque en el análisis de proyecciones de incertidumbres. Además, las simulaciones de GCs son menos sesgadas en climas templados húmedos que en climas secos (Breinl et al., 2017; Urdiales et al., 2018). Por tal motivo, los GCs son prometedores para el mejoramiento del pronóstico de la precipitación en Los Andes tropicales y además su implementación tiene un bajo costo computacional. Hasta la actualidad, se pueden enumerar varios GCs en la categoría de enfoques estocásticos-estadísticos, como WXGEN (Sharpley et al., 1990), CLIGEN (Lobo et al., 2015), LARS-WG (Semenov et al., 2002), WeaGETS (Chen et al., 2012), KnnCAD (Gaur et al., 2015; King et al., 2014, 2015), y el GC utilizado por la Oficina Meteorológica del Reino Unido (UKCP09) (Christierson et al., 2012). Una alternativa para mejorar la estimación de la precipitación diaria es la incorporación de modelos lineales generalizados (MLG) con regresiones o funciones logísticas (Kim et al., 2012) a los GCs, ya que es un método útil cuando consideramos variables escalares y/o binarias y para cuantificar los pronósticos de precipitación que son claramente no Gaussianos (Wilks, 2009). El uso temprano de MLG fue realizado por Stern et al., (1984) y consecutivamente utilizado por Chandler, (2005) y Yang et al., (2005). Posteriormente un enfoque de MLG en GCs fue usado por Verdin et al., (2014) y Furrer et al., (2008) quienes argumentan que se obtiene una mejora sustancial en la estimación de precipitación extrema a partir de varios enfoques en la distribución de la intensidad de precipitación (por ejemplo, técnicas híbridas que permiten pronosticar intensidades altas y moderadas con una distribución Gamma y baja intensidad mediante una distribución de Pareto).

En este contexto, nuestro objetivo es analizar las posibilidades de éxito de realizar un pronóstico de ocurrencia de precipitación mediante un GC diario en una gradiente altitudinal de una zona andina del sur del Ecuador, a partir de las observaciones del comportamiento atmosférico y las relaciones con variables (ocurrencia del día anterior $\left(\mathrm{K}_{\mathrm{t}}\right.$ 1), las temperaturas máximas y mínimas del día anterior $\left(T x_{t-1}, T n_{t-1}\right.$, respectivamente)) y covariables (anomalías de la Temperatura mensual Superficial del Mar de El NiñoOscilación del Sur (El Niño 1+2 y El Niño 3.4 respectivamente)) intrínsecas en el sistema climático. Si bien en la literatura se encuentra que para alcanzar una buena calidad predictiva de precipitación la implementación de los GCs se debe realizar con series temporales de observaciones sobre los 10 años (Richardson, 2000; Soltani et al., 2003), en el presente estudio el objetivo no se centra en alcanzar una alta precisión sino en (i) identificar las potencialidades de tal herramienta de pronóstico para una cuenca andina con alta variabilidad y (ii) levantar y/o rechazar hipótesis que permitan entender el funcionamiento del sistema. 


\section{2. Área de estudio y datos climáticos}

El área de estudio se ubica en el flanco oriental de la Cordillera Occidental de los Andes y cubre un rango altitudinal de entre los 2500 y 4000 m s.n.m. Cuatro de los cinco puntos de monitoreo se encuentran ubicados a diferentes elevaciones y distancias (aproximadamente $5 \mathrm{~km}$ entre los dos sitios más cercanos y $24 \mathrm{~km}$ entre los sitios más distantes), es parte del Observatorio Ecohidrológico Quinuas, el cual se encuentra en la cabecera de la cuenca del río Paute, la cual es afluente del Río Amazonas. Aquí se encuentran los sitios de monitoreo: (i) Chirimachay; (ii) La Virgen; (iii) Toreadora y (iv) Balzay. La otra estación restante se encuentra en la cabecera del río Machángara y se denomina (v) Labrado. Los datos detallados de las estaciones se encuentran en la Tabla 1 y su ubicación en la Figura 1.

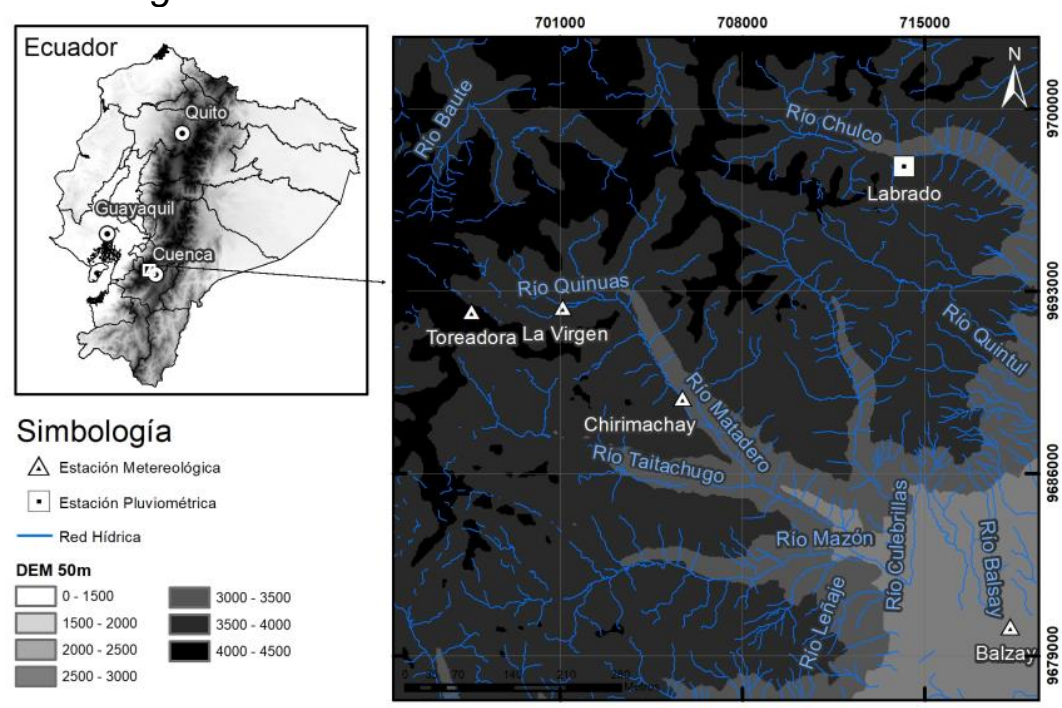

Figura 1. Distribución espacial de estaciones.

Las cinco estaciones tienen datos históricos diarios de ocurrencia de precipitación $\left(\mathrm{K}_{\mathrm{t}}\right)$. Adicionalmente, cuatro de ellas poseen datos de temperatura máxima (Tx) y temperatura mínima (Tn) (Tabla 1). Finalmente, las series temporales de los índices ENOS (Niño 1+2 y Niño 3.4) abarcan las anomalías mensuales desde enero de 1950 a febrero de 2017 y fueron obtenidas del Departamento de Administración Atmosférica y Oceánica de los Estados Unidos (NOAA por sus siglas en inglés; http://www.esrl.noaa.gov/psd/data/ climateindices/list/).

Tabla 1. Registro de datos históricos de precipitación.

\begin{tabular}{|c|c|c|c|c|c|c|c|}
\hline \multirow{2}{*}{ Estación } & \multirow{2}{*}{ Latitud } & \multirow{2}{*}{ Longitud } & \multirow{2}{*}{$\begin{array}{l}\text { Altitud } \\
\text { (m } \\
\text { s.n.m) }\end{array}$} & \multirow{2}{*}{$\begin{array}{l}\text { Tipo de } \\
\text { Estación }\end{array}$} & \multicolumn{2}{|c|}{ Datos Disponibles } & \multirow{2}{*}{$\begin{array}{c}\text { Datos } \\
\text { perdidos } \\
\text { (\%) }\end{array}$} \\
\hline & & & & & Inicio & Fin & \\
\hline Labrado & $2^{\circ 043^{\prime}}$ & 790' & 3434 & nétrica & $01 / 01 / 1964$ & $29 / 09 / 2011$ & 7 \\
\hline Balzay & $2^{\circ} 53^{\prime}$ & $79^{\circ} 02^{\prime}$ & 2603 & Meteorológica & $01 / 01 / 2014$ & $31 / 02 / 2017$ & 0 \\
\hline Chirimachay & $2^{\circ} 49^{\prime}$ & $79^{\circ} 9^{\prime}$ & 3298 & Meteorológica & $01 / 01 / 2014$ & $31 / 02 / 2017$ & 0 \\
\hline La Virgen & $2^{\circ} 47^{\prime}$ & 791' & 3626 & Meteorológica & $01 / 01 / 2014$ & $31 / 02 / 2017$ & 0 \\
\hline Toreadora & $2^{\circ} 47^{\prime}$ & 791' & 3955 & Meteorológica & $01 / 01 / 2014$ & $31 / 02 / 2017$ & 0 \\
\hline
\end{tabular}

\section{Metodología}

La ocurrencia de precipitación fue modelada con una cadena de Markov de primer orden. La probabilidad condicional de la ocurrencia de precipitación en cualquier día solo depende de la precipitación del día previo. La ocurrencia de precipitación es tratada como 
una variable binaria $K_{t}=1$ para día con una precipitación mayor a cero (lluvioso) y $K_{t}=0$ por día sin precipitación (seco). La cadena de Markov de primer orden es caracterizada mediante las probabilidades de transición en la Ecuación 1 y Ecuación 2:

$$
\begin{aligned}
& p_{01}(t)=\operatorname{Pr}\left(K_{t}=1 \quad \mid K_{t-1}=0\right) \\
& p_{11}(t)=\operatorname{Pr}\left(K_{t}=1 \quad \mid K_{t-1}=1\right)
\end{aligned}
$$

Donde, $\operatorname{Pr}$ denota probabilidad. $p_{01}$ representa la probabilidad de evento de lluvia si el día anterior fue seco y $p_{11}$ explica la probabilidad de evento de lluvia si el día anterior fue lluvioso. El modelo para la ocurrencia de precipitación es un MLG binomial (p. ej., variables 0 o 1). El valor esperado de una distribución binomial con un único ensayo (también llamado distribución de Bernoulli) es la probabilidad de éxito subyacente, es decir, en nuestro caso la probabilidad de precipitación. EI MLG binomial con función logística fue usado para relacionar covariables a la ocurrencia de precipitación. Nuestra variable primaria es la ocurrencia de lluvia en el día anterior $K_{t-1}$, de modo que actualmente tenemos una cadena de Markov de primer orden. Usamos como covariables la anomalía mensual de El Niño $1+2$ y El Niño 3.4; para luego extrapolarlos diariamente. Además, los valores históricos de temperatura máxima del día previo $\left(T x_{t-1}\right)$ y temperatura mínima del día anterior $\left(\mathrm{Tn}_{\mathrm{t}-1}\right)$. También se pueden introducir covariables adicionales, como un ciclo estacional u otros índices climáticos. La siguiente expresión matemática se utilizó para obtener las probabilidades condicionales (Ecuación 3); en este caso Covariable hace referencia a El Niño 1+2, El Niño 3.4, $\mathrm{TX}_{\mathrm{t}-1}$ y $\mathrm{Tn}_{\mathrm{t}-1}$. La estimación de los parámetros de la Ecuación 3 se realizó por el método de momentos.

$$
p_{1}=\frac{\exp ^{\wedge}\left(\beta_{0}+\beta_{1} K_{t-1}+\beta_{2} \text { Covariable }\right)}{1+\exp ^{\wedge}\left(\beta_{0}+\beta_{1} K_{t-1}+\beta_{2} \text { Covariable }\right)}
$$

En este caso, la notación ha sido ligeramente modificada. El valor calculado en la Ecuación 3 produce la probabilidad de tener un día húmedo. Los valores específicos de $\mathrm{K}_{\mathrm{t}} 1$ (0 o 1) nos permiten reproducir las probabilidades condicionales de una cadena de Markov. Dada la no estacionalidad de los regímenes de precipitación, el modelo fue adecuado para obtener parámetros mensuales. Para determinar si se produjo precipitación en el día simulado, se generó un número aleatorio uniforme (v1). Dado esto, cuando $p_{1}>\mathrm{v} 1$, se obtiene un evento de lluvia, de lo contrario se registra como un día seco o no lluvioso. La simulación estocástica de ocurrencia de precipitación se describe en la Figura 2, la cual fue programada en R Studio versión 3.3, mediante el enfoque glm con función logit y la generación de números aleatorios con la función runif.

\section{Resultados}

Probabilidades de ocurrencia de precipitación y modelo lineal generalizado (MLG) para el pronóstico diario.

La Figura 3, describe las probabilidades de transición de La Virgen. Esta representación se realizó para todas las estaciones, las cuales mostraron similar comportamiento, se explica que El Niño 3.4 influye a la variabilidad climática intranual e interanual de esta zona altoandina. Por ejemplo, en abril y mayo las probabilidades de un evento de precipitación son menores al $50 \%$, tales probabilidades se presentan cuando El Niño 3.4 tiene valores de anomalías extremos concretamente menores a cero (La Niña), esto es más notorio en la probabilidad de un día lluvioso previo a un día seco $\left(p_{01}\right)$. Asimismo, las probabilidades de ocurrencia de lluvia aumentan considerablemente cuando El Niño 3.4 toma valores de anomalías mayores a dos (El Niño), lo cual denota una disminuida variabilidad interanual de junio a diciembre tanto para las probabilidades $p_{01}$ y $p_{11}$. 


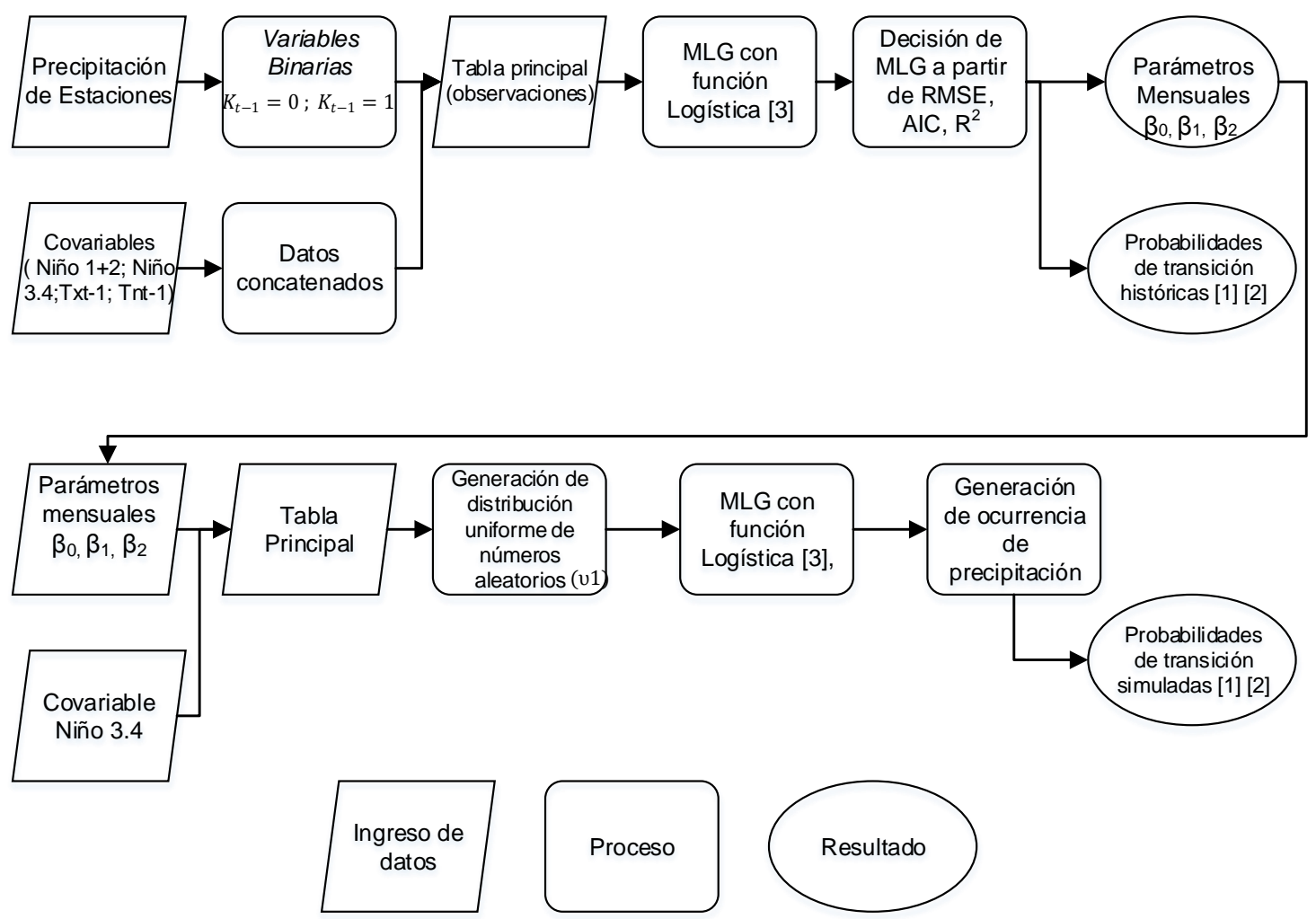

Figura 2. Simulación estocástica diaria de la ocurrencia de precipitación. Números en corchetes hacen referencia a las ecuaciones en el texto.
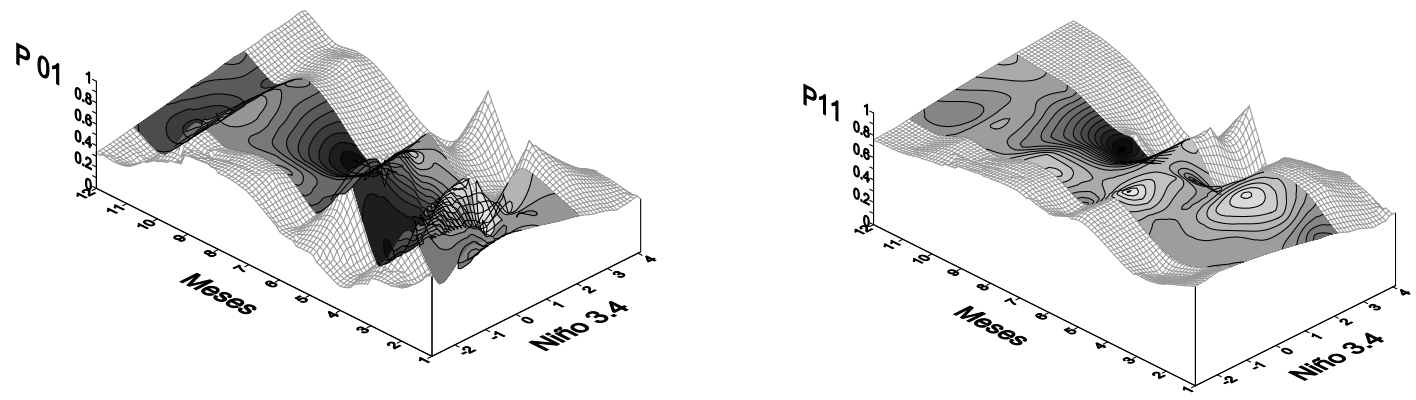

Figura 3. Probabilidades de transición diaria El Niño 3.4. $p_{01}$ : probabilidad de evento de lluvia si el día anterior fue seco. $p_{11}$ : probabilidad de evento de lluvia si el día anterior fue lluvioso. Isolíneas a partir de datos observados, pixel o malla a partir de datos simulados.

En la Tabla 2 se presentan los MLG mensuales con funciones logísticas, los meses escogidos son los cuales presentan notable mejora en el ajuste del pronóstico. Esto fue identificado mediante el coeficiente de determinación $\left(R^{2}\right)$, el Criterio de Información Akaike (AIC por sus siglas en inglés) (Akaike, 1987) y el error cuadrático medio (RMSE). Además, se muestran en mayor cantidad los meses de temporadas lluviosas (febrero a mayo y de octubre a noviembre en todas las estaciones) ya que asumimos que en dichos meses deberíamos tener más éxito en el pronóstico de ocurrencia; se obtienen resultados con menor ajuste para otros meses de temporadas secas (p. ej., enero, junio, julio, agosto).

La Figura 4 presenta un compendio mensual de las probabilidades de transición diarias de un evento de lluvia previo a un día seco $\left(p_{01}\right)$ y las probabilidades de transición a escala diaria de un evento de precipitación si el día anterior fue lluvioso $\left(p_{11}\right)$. De manera general se observa que $p_{01}$ presenta una estacionalidad más notoria que $p_{11}$. Para $p_{01}$ la diferencia visible cuando relacionamos la media de probabilidad mensual y el gradiente 
altitudinal, por ejemplo, las probabilidades observadas de agosto en Chirimachay oscilan de 0,22 a 0,61 con $\mu=0,37$; mientras que en La Virgen la $\mu$ observada $=0,60$ y para La Toreadora $\mu$ observada $=0,51$; por lo cual no existe un patrón en estas tres estaciones con respecto a $p_{01}$. En la Figura 4 la probabilidad media de un día lluvioso posterior a otro lluvioso $\left(p_{11}\right)$ sobrepasa el $70 \%$ en todos los meses y en las 3 estaciones más elevadas del gradiente. Esto demuestra que un evento de lluvia es más probable que suceda si el día anterior fue lluvioso. Sin embargo, $p_{11}$ tiene una estacionalidad compleja de visualizar, o no existe dicha variabilidad interanual; hay meses de los años simulados donde las probabilidades son menores a 0,5 (abril y mayo). Adicionalmente, las probabilidades de transición $p_{11}$ simuladas en julio en Chirimachay tienen un rango de 0,37 a 0,95 con $\mu=$ 0,87 y para La Virgen de 0,68 a 0,86 con $\mu=0,80$ y por último en La Toreadora las probabilidades en dicho mes van desde 0,77 a 0,89 con $\mu=0,79$. Finalmente, en octubre y noviembre el rango de dispersión es más cercano a la máxima probabilidad sobre todo en la estación Chirimachay. Por lo tanto, asumimos que en mayor medida las máximas probabilidades de precipitación se observan en los 3298 m s.n.m en una temporada lluviosa.

Tabla 2. Estadígrafos de Modelos Lineales Generalizados (MLG). Se presentan las regresiones logísticas mensuales donde es significativa la incorporación de alguna variable o covariable. Regresiones marcadas en gris denotan mejor ajuste en el pronóstico. $\mathrm{K}_{\mathrm{t}-1}$ : Ocurrencia del día anterior. Tn t-1: Temperatura mínima del día anterior. Niño 3.4: índice de la anomalía de la temperatura superficial del mar de la región 3.4.

\begin{tabular}{|c|c|c|c|c|c|c|c|c|c|c|}
\hline \multirow[t]{2}{*}{ Estación } & \multirow[t]{2}{*}{ Mes } & \multicolumn{3}{|c|}{ MLG con $\mathrm{K}_{\mathrm{t}-1}$} & \multicolumn{3}{|c|}{$\begin{array}{c}\text { MLG con } \mathrm{K}_{\mathrm{t}-1} \text { y el Niño } \\
3.4\end{array}$} & \multicolumn{3}{|c|}{ MLG con $\mathrm{K}_{\mathrm{t}-1}$ y Tn $\mathrm{t}-1$} \\
\hline & & AIC & $\mathbf{R}^{2}$ & RMSE & AIC & $\mathbf{R}^{2}$ & RMSE & AIC & $\mathbf{R}^{2}$ & RMSE \\
\hline \multirow[t]{2}{*}{ Labrado } & Ene & 1524,1 & 0,21 & 1,05 & 1516,5 & 0,23 & 1,05 & - & - & - \\
\hline & Feb & 1318,7 & 0,13 & 1,01 & 1309,4 & 0,15 & 1 & - & - & - \\
\hline \multirow{3}{*}{ Balzay } & Mar & 112,2 & 0,17 & 1,08 & - & - & - & - & - & - \\
\hline & May & - & - & - & - & - & - & 71,82 & 0,25 & 1,06 \\
\hline & Jun & 75,9 & 0,14 & 1,09 & - & - & - & 70,34 & 0,24 & 0,91 \\
\hline \multirow{4}{*}{ Chirimachay } & Feb & 105,4 & 0,16 & 0,95 & - & - & - & 98,3 & 0,27 & 0,90 \\
\hline & Abr & 112,9 & 0,44 & 0,96 & 110,8 & 0,47 & 0,94 & - & - & - \\
\hline & Jul & 132,8 & 0,36 & 1,02 & 104,7 & 0,57 & 0,89 & - & - & - \\
\hline & Ago & 139,9 & 0,30 & 1,05 & 102,45 & 0,56 & 0,79 & - & - & - \\
\hline \multirow{4}{*}{ La Virgen } & Feb & 83,2 & 0,25 & 0,97 & - & - & - & 75,91 & 0,38 & 0,91 \\
\hline & Abr & 62,2 & 0,67 & 0,80 & 56,8 & 0,72 & 0,75 & - & - & - \\
\hline & Oct & 143,3 & 0,20 & 1,08 & - & - & - & 128,6 & 0,35 & 1,01 \\
\hline & Sep & 106,3 & 0,19 & 1,01 & - & - & - & 100,7 & 0,28 & 0,97 \\
\hline \multirow{5}{*}{ Toreadora } & Feb & 99,1 & 0,27 & 0,92 & - & - & - & 75,91 & 0,38 & 0,91 \\
\hline & Abr & 110,3 & 0,47 & 0,94 & 107,5 & 0,51 & 0,92 & - & - & - \\
\hline & May & 94,9 & 0,01 & 0,86 & 91,2 & 0,1 & 0,83 & - & - & - \\
\hline & Oct & 156,8 & 0,12 & 1,01 & - & - & - & 154,7 & 0,16 & 0,99 \\
\hline & Nov & 153,3 & 0,26 & 1,07 & 152,1 & 0,27 & 1,07 & & & \\
\hline
\end{tabular}

Probabilidades de ocurrencia de precipitación a escala del gradiente altitudinal. Relación temperatura mínima del día anterior con la longitud de días secos y húmedos

Los patrones de longitud de días secos previos a un evento de precipitación fueron sustancialmente diferentes a más altas elevaciones e inversamente relacionados con el descenso de la temperatura, como se destacan en la Figuras $5 b, 5 d, 5 f$, $5 h$. Además, la máxima longitud de días con precipitación previos a un día seco es relativamente constante en el gradiente altitudinal ya que oscilan entre 12 días, excepto para La Virgen que fue de 22 días (Figuras 4a, 4c, 4e, 4g).

\section{Discusiones y conclusiones}

La metodología de simulación estocástica del componente de ocurrencia de precipitación en la presente investigación ha sido capaz de reproducir eficazmente la 

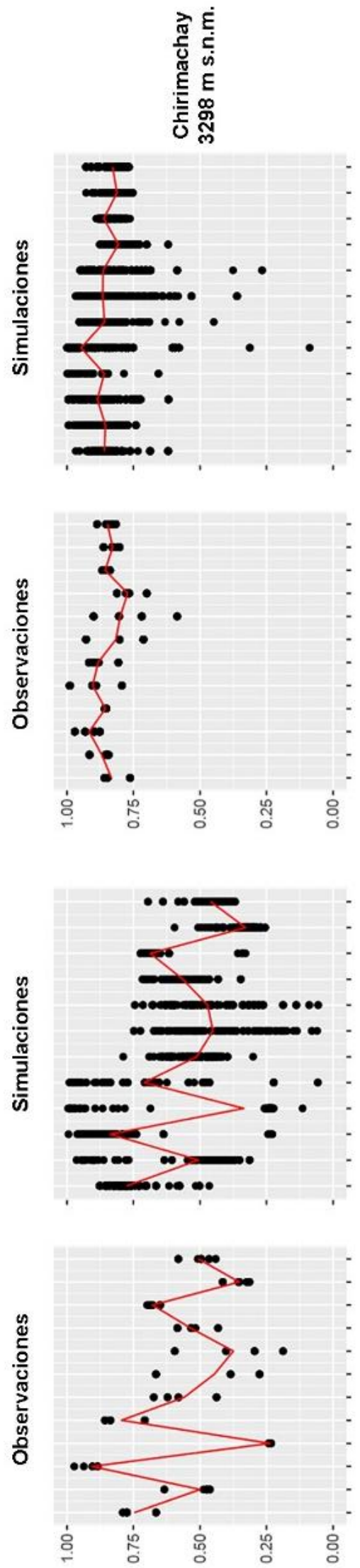
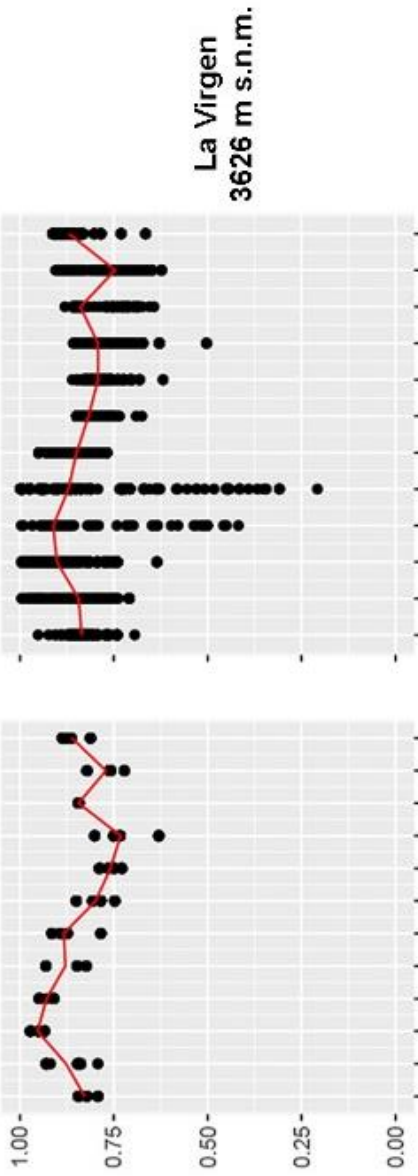

$\left(\mathfrak{u}_{d}\right)$ pep!!!qeqodd
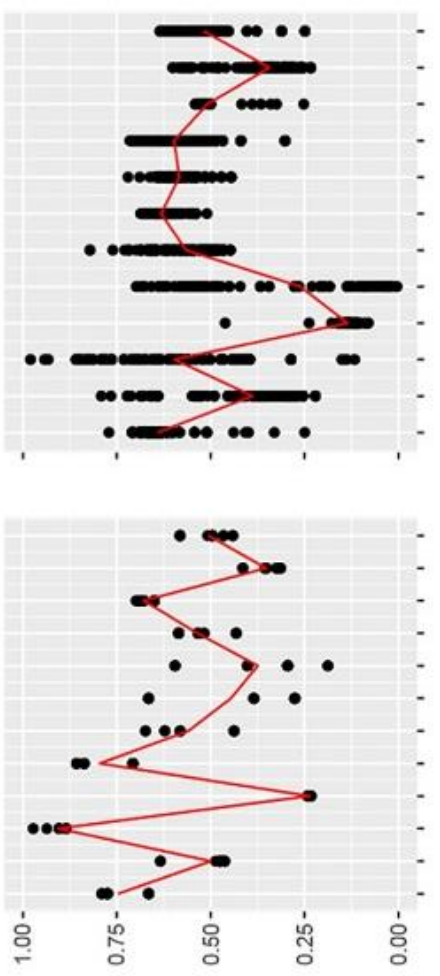
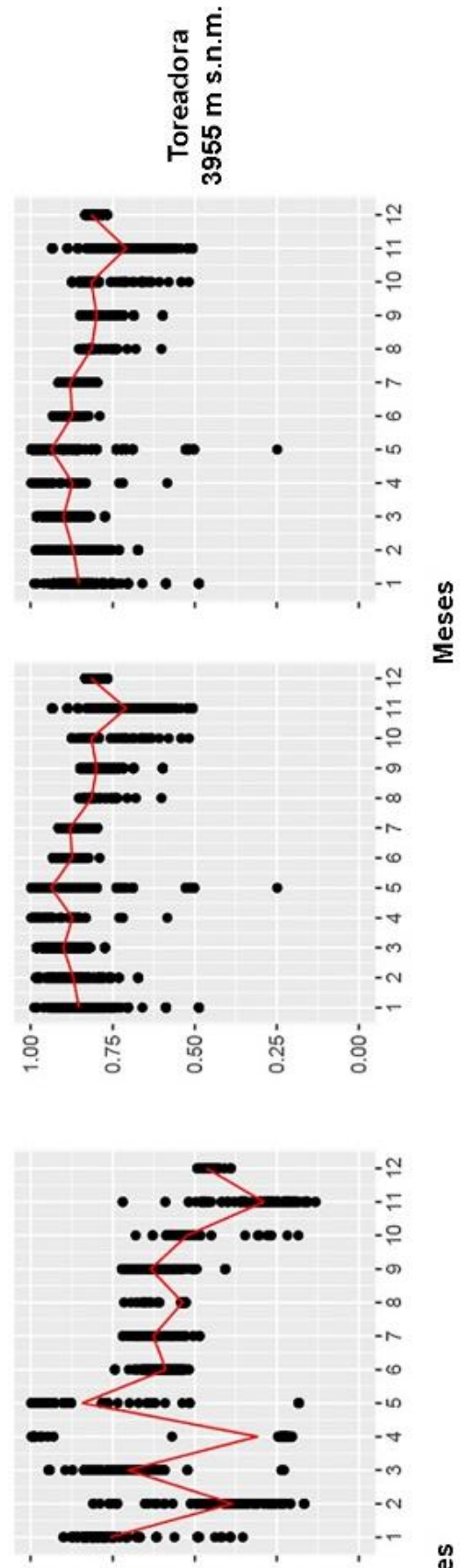

岕

Figura 4. Probabilidades de transición diarias para un evento de precipitación previo a un día seco $\left(p_{01}\right)$ y probabilidades de transición diarias de un día lluvioso previo a un día lluvioso $\left(p_{11}\right)$. Línea roja representa la media de las probabilidades diarias en cada mes. 
variabilidad interanual e intranual, a pesar que no se realizó una validación de nuestros resultados por motivo de la extensión temporal de nuestros datos. Sin embargo, con la estación de Labrado, la cual tiene la mayor cantidad de observaciones se encontraron similares resultados que con las otras estaciones restantes, es decir aporte significativo en el pronóstico de ocurrencia de la variable $\mathrm{K}_{\mathrm{t}-1}$ y la covariable el Niño 3.4. Además, concluimos que el Niño 3.4 influye en la dinámica temporal de las probabilidades de transición en zonas montañosas de Ecuador, lo cual concuerda con estudios anteriores (Vicente-Serrano et al., 2017). Se demuestra que las probabilidades de transición $p_{01}$ tienen una estacionalidad más marcada que $p_{11}$; es decir, $p_{11}$ toma muchas veces más valores cercanos a uno para todo el año.
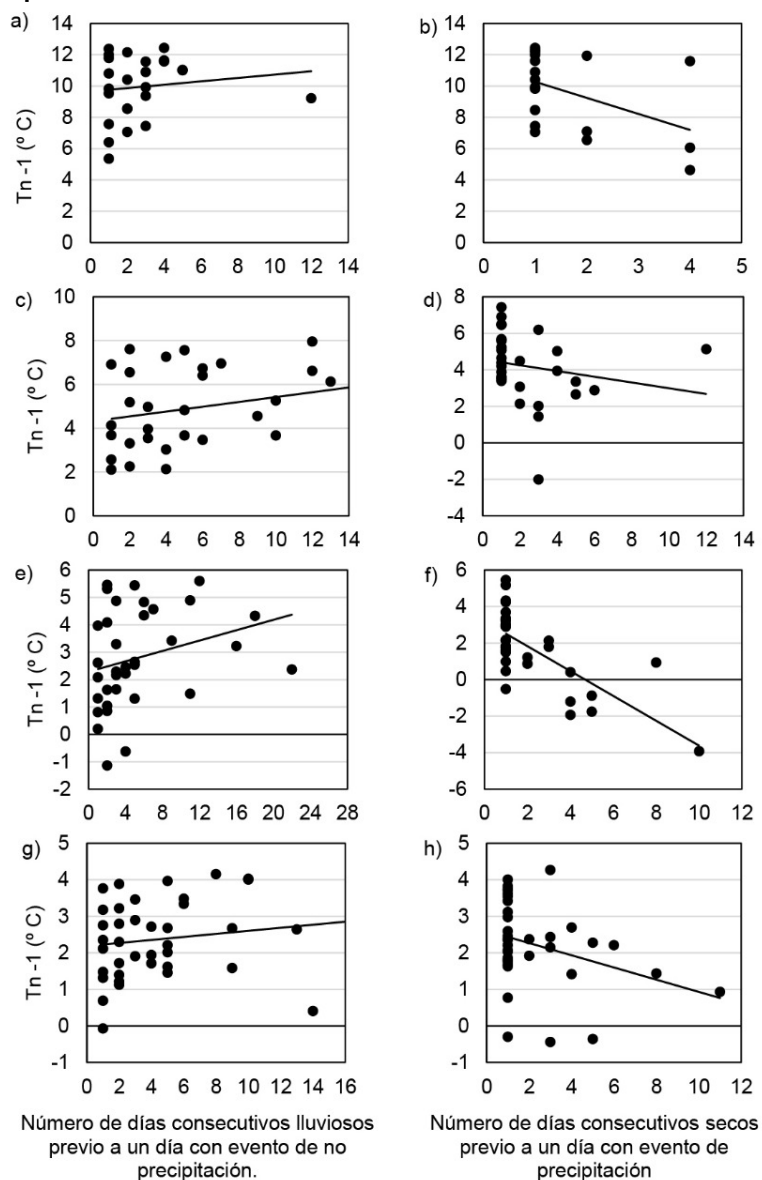

Figura 5. Número de días consecutivos con precipitación o secos a partir de observaciones para octubre y noviembre. $\mathrm{Tn}_{\mathrm{t}-1}$ denota la temperatura mínima del día anterior al evento (día lluvioso o día seco). a) y b) Balzay, c) y d) Chirimachay, e) y f) La Virgen, g) y h) La Toreadora.

En nuestro estudio se encontró que la temperatura mínima del día anterior $\left(\operatorname{Tn}_{\mathrm{t}-1}\right)$ es significativa para la estimación de la probabilidad de ocurrencia de precipitación. Este es un hallazgo importante porque nos permite inferir en el comportamiento del sistema climático y en la influencia del gradiente altitudinal. Por ello, futuros estudios deberían poner a prueba las siguientes hipótesis: i) ¿Mejoramos el pronóstico de ocurrencia de lluvia al aumentar el orden de la cadena de Markov? Es decir, ¿el evento de precipitación puede depender no solo de la temperatura mínima del día anterior sino de las $\operatorname{Tn}_{\mathrm{t}-1}$ de varios días previos?,ii) ¿Una alta probabilidad de lluvia no solo está condicionada a la temperatura mínima del día previo en el propio sitio sino a dicha temperatura de uno o más sitios en el gradiente altitudinal? Además, reforzamos la hipótesis de Tapia (2016), quien demostró que la precipitación máxima anual en la gradiente se produce entre los 3000 y $3300 \mathrm{~m}$ s.n.m., lo cual nos da indicio que el GC utilizado es prometedor para pronosticar espacialmente la ocurrencia de precipitación en una zona montañosa. 
La deficiencia más notoria del modelo fue que en meses secos el ajuste del pronóstico disminuye considerablemente si comparamos con meses húmedos, razón por la cual concluimos que un modelo ajustado para todos los meses no logra abarcar la estacionalidad de la ocurrencia de eventos de baja frecuencia, por lo cual se debería explorar la utilización de varios modelos adaptados para distintas condiciones de precipitación. Finalmente, el trabajo a futuro que está por explorar es realizar un ensamble del presente GC con Sistemas de Pronóstico Global (GFS sus siglas en inglés), y así disminuir la incertidumbre en el pronóstico de la ocurrencia de precipitación a escala diaria.

Este es el primer estudio reportado en Los Andes del Norte que utiliza un GC para realizar pronósticos diarios de ocurrencia de precipitación. El principal hallazgo es que el Niño 3.4 y la $T n_{t-1}$ en un esquema de GLM mejora la representación del proceso de ocurrencia de precipitación (es decir, permite un mejor pronóstico de las probabilidades de transición). Además, se han podido levantar varias hipótesis sobre el funcionamiento climático de las cuencas andinas con relación a las cuales sientan las bases para continuar las líneas de investigación de pronóstico y de física de procesos en el futuro.

\section{Agradecimientos}

Este estudio fue posible gracias a los proyectos "Identificación de los procesos hidrometeorológicos que desencadenan crecidas, a partir de la información suministrada por un radar de precipitación", financiado por la Dirección de Investigación de la Universidad de Cuenca (DIUC) y ETAPA EP y "Optimización de muestreo espacial de la precipitación con geo estadística multivariada", financiado por la DIUC. Los autores agradecen las valiosas contribuciones de Victoria Madrid (Figura 1) y a los dos revisores anónimos cuyos comentarios detallados mejoraron en gran medida este documento.

\section{Bibliografía}

Akaike, H. (1987). Factor analysis and AIC. Psychometrika, 52(3), 317-332.

Avilés, A., Célleri, R., Paredes, J., \& Solera, A. (2015). Evaluation of Markov Chain Based Drought Forecasts in an Andean Regulated River Basin Using the Skill Scores RPS and GMSS. Water Resources Management, 1949-1963. http://doi.org/10.1007 /s11269-015-0921-2

Ballari, D., Giraldo, R., Campozano, L., \& Samaniego, E. (2018). Spatial functional data analysis for regionalizing precipitation seasonality and intensity in a sparsely monitored region: Unveiling the spatio-temporal dependencies of precipitation in Ecuador. International Journal of Climatology.

Bauer, P., Thorpe, A., \& Brunet, G. (2015). The quiet revolution of numerical weather prediction. Nature, 525(7567), 47-55. http://doi.org/10.1038/nature14956

Breinl, K., Di Baldassarre, G., Girons Lopez, M., Hagenlocher, M., Vico, G., \& Rutgersson, A. (2017). Can weather generation capture precipitation patterns across different climates, spatial scales and under data scarcity? Scientific Reports, 7(1), 5449. http://doi.org/10.1038/s41598-017-05822-y

Buytaert, W., Célleri, R., De Bièvre, B., Cisneros, F., Wyseure, G., Deckers, J., \& Hofstede, R. (2006). Human impact on the hydrology of the Andean páramos. Earth-Science Reviews, 79(1), 53-72.

Caloiero, T., Sirangelo, B., Coscarelli, R., \& Ferrari, E. (2016). An analysis of the occurrence probabilities of wet and dry periods through a stochastic monthly rainfall model. Water (Switzerland), 8(2). http://doi.org/10.3390/w8020039

Campozano, L., Ballari, D., \& Celleri, R. (2014). Imágenes TRMM para identificar patrones de precipitación e índices ENSO en Ecuador. MASKANA, I+D+ingeniería 2014, (May), 185-191.

Celleri, R., Willems, P., Buytaert, W., \& Feyen, J. (2007). Space-time rainfall variability in the Paute basin, Ecuadorian Andes. Hydrological Processes, 21(24), 3316-3327. 
Chandler, R. E. (2005). On the use of generalized linear models for interpreting climate variability. Environmetrics, 16(7), 699-715. http://doi.org/10.1002/env.731

Chen, J., Brissette, F. P., \& Leconte, R. (2012). WeaGETS-a Matlab-based daily scale weather generator for generating precipitation and temperature. Procedia Environmental Sciences, 13, 2222-2235.

Cherubini, T., Ghelli, A., \& Lalaurette, F. (2002). Verification of Precipitation Forecasts over the Alpine Region Using a High-Density Observing Network. Weather and Forecasting, 17(2), 238-249. http://doi.org/10.1175/15200434(2002)017<0238:VOPFOT>2.0.CO;2

Chou, S., Bustamante, J., \& Gomes, J. (2005). Evaluation of Eta model seasonal precipitation forecasts over South America. Nonlinear Processes in Geophysics, 12(4), 537-555.

Christierson, B. v, Vidal, J.-P., \& Wade, S. D. (2012). Using UKCP09 probabilistic climate information for UK water resource planning. Journal of Hydrology, 424, 48-67.

De Biévre, Alvarado, A., Timbe, L., Célleri, R., \& Feyen, J. (2003). Night irrigation reduction for water saving in medium-sized systems.

Espinoza, J. C., Ronchail, J., Vauchel, P. (2009). Spatio-temporal rainfall variability in the Amazon basin countries (Brazil, Peru, Bolivia, Colombia, and Ecuador). International Journal of Climatology, 29(11), 1574-1594.

Francou, B., Vuille, M., Favier, V., \& Cáceres, B. (2004). New evidence for an ENSO impact on low-latitude glaciers: Antizana 15, Andes of Ecuador, $0^{\circ} 28^{\prime} \mathrm{S}$. Journal of Geophysical Research Atmospheres, 109(18), 1-17. http://doi.org/10.1029/2003JD004484

Furrer, E. M., \& Katz, R. W. (2008). Improving the simulation of extreme precipitation events by stochastic weather generators. Water Resources Research, 44(12), 1-13. http://doi.org/10.1029/2008WR007316

Gaur, A., \& Simonovic, S. P. (2015). Towards reducing climate change impact assessment process uncertainty. Environmental Processes, 2(2), 275-290.

González, J., \& Lajara, J. (2015). Errors Analysis in extreme rainfall recording due to tipping bucket rain gauge performance. The Netherlands.

Kalnay, E. (2003). Atmospheric modeling, data assimilation and predictability. Cambridge university press.

Kim, Y., Katz, R. W., Rajagopalan, B., Podestá, G. P., \& Furrer, E. M. (2012). Reducing overdispersion in stochastic weather generators using a generalized linear modeling approach. Climate research, 53(1), 13-24.

King, L. M., McLeod, A. I., \& Simonovic, S. P. (2014). Simulation of historical temperatures using a multi-site, multivariate block resampling algorithm with perturbation. Hydrological Processes, 28(3), 905-912.

King, L. M., McLeod, A. I., \& Simonovic, S. P. (2015). Improved weather generator algorithm for multisite simulation of precipitation and temperature. JAWRA Journal of the American Water Resources Association, 51(5), 1305-1320.

Kumar, S. (2015). Spatial Distribution of Rainfall with Elevation in Satluj River Basin: 19862010, Himachal Pradesh, India. International Letters of Chemistry, Physics and Astronomy, 57, 163. http://doi.org/10.18052/www.scipress.com/ILCPA.57.163

Lobo, G. P., Frankenberger, J. R., Flanagan, D. C., \& Bonilla, C. A. (2015). Evaluation and improvement of the CLIGEN model for storm and rainfall erosivity generation in Central Chile. Catena, 127, 206-213.

Mishra, A. K., \& Desai, V. R. (2005). Drought forecasting using stochastic models. Stochastic Environmental Research and Risk Assessment, 19(5), 326-339. http://doi.org/10.1007/s00477-005-0238-4

Muñoz, P., Célleri, R., \& Feyen, J. (2016). Effect of the resolution of tipping-bucket rain gauge and calculation method on rainfall intensities in an andean mountain gradient. Water (Switzerland), 8(11). http://doi.org/10.3390/w8110534 
Ochoa, B. F., Buytaert, W., ... Arias, S. (2016). Impacts of land use on the hydrological response of tropical Andean catchments. Hydrological Processes, 4089(August), 4074-4089. http://doi.org/10.1002/hyp. 10980

Richardson, C. W. (2000). Data requirements for estimation of Weather Generation parameters. St. Joseph, Mich: ASABE. http://doi.org/10.13031/2013.2983

Rollenbeck, R., \& Bendix, J. (2011). Rainfall distribution in the Andes of southern Ecuador derived from blending weather radar data and meteorological field observations. Atmospheric Research, 99(2), 277-289.

Ropelewski, C. F., \& Halpert, M. S. (1996). Quantifying southern oscillation-precipitation relationships. Journal of climate, 9(5), 1043-1059.

Rossel, F., Le Goulven, P., \& Cadier, E. (1999). Areal distribution of the influence of ENSO on the annual rainfall in Ecuador [El Nino/Southern oscillation]. Revue des Sciences de l'Eau (France).

Semenov, M. a, \& Barrow, E. M. (2002). A Stochastic Weather Generator for Use in Climate Impact Studies. User Manual, Hertfordshire, UK, (August), 0-27.

Sharpley, A. N., \& Williams, J. R. (1990). EPIC-Erosin/productivity Impact Calculator: 1. Model Documentation. USDA.

Shi, H., Li, T., Wei, J., Fu, W., \& Wang, G. (2016). Spatial and temporal characteristics of precipitation over the Three-River Headwaters region during 1961-2014. Journal of Hydrology: Regional Studies, 6, 52-65. http://doi.org/10.1016/j.ejrh.2016.03.001

Sokol, Z., \& Bližňák, V. (2009). Areal distribution and precipitation-altitude relationship of heavy short-term precipitation in the Czech Republic in the warm part of the year. Atmospheric Research, 94(4), 652-662. http://doi.org/10.1016/j.atmosres.2009.03.001

Soltani, A., \& Hoogenboom, G. (2003). Minimum data requirements for parameter estimation of stochastic weather generators. Climate Research, 25, 109-119. http://doi.org/10.3354/cr025109

Srikanthan, R., \& McMahon, T. a. (2001). Stochastic generation of annual, monthly and daily climate data: A review. Hydrology and Earth System Sciences, 5(4), 653-670. http://doi.org/10.5194/hess-5-653-2001

Stern, R. D., \& Coe, R. (1984). A model fitting analysis of daily rainfall data. J. Roy. Stat. Soc. $A, 147(1), 1-34$.

Tapia, J. (2016). Estructura de la precipitación y su variabilidad espacio-temporal a lo largo de una gradiente altitudinal. Tesis de Licenciatura. Universidad de Cuenca. Recuperado a partir de http://dspace.ucuenca.edu.ec/handle/123456789/24348

Ulloa, J., Ballari, D., Campozano, L., \& Samaniego, E. (2017). Two-Step Downscaling of Trmm 3b43 V7 Precipitation in Contrasting Climatic Regions With Sparse Monitoring: The Case of Ecuador in Tropical South America. Remote Sensing, 9(7), 758.

Urdiales, D., Meza, F., Gironás, J., \& Gilabert, H. (2018). Improving Stochastic Modelling of Daily Rainfall Using the ENSO Index: Model Development and Application in Chile. Water, 10(2), 145.

Verdin, A., Rajagopalan, B., Kleiber, W., \& Katz, R. W. (2014). Coupled stochastic weather generation using spatial and generalized linear models. Stochastic Environmental Research and Risk Assessment, (Richardson 1981), 347-356. http://doi.org/10.1007/s00477-014-0911-6

Vicente-Serrano, S. M., Aguilar, E., Nieto, R. (2017). The complex influence of ENSO on droughts in Ecuador. Climate Dynamics, 48(1-2), 405-427. http://doi.org/10.1007/s00382-016-3082-y

Vuille, M., \& Werner, M. (2005). Stable isotopes in precipitation recording South American summer monsoon and ENSO variability: observations and model results. Climate Dynamics, 25(4), 401-413.

Walker, G. T. (1924). Correlations in seasonal variations of weather. I. A further study of world weather. Mem. Indian Meteorol. Dep., 24, 275-332. 
Wan, H., Zhang, X., \& Barrow, E. M. (2005). Stochastic modelling of daily precipitation for Canada. Atmosphere-Ocean, 43(1), 23-32. http://doi.org/10.3137/ao.430102

Wastl, C., \& Zängl, G. (2010). Mountain-valley precipitation differences in the northern Alps: An exemplary high-resolution modeling study. Meteorology and Atmospheric Physics, 108(1), 29-42. http://doi.org/10.1007/s00703-010-0083-y

Wilks, D. S. (2009). Extending logistic regression to provide full-probability-distribution MOS forecasts, 368(March), 361-368. http://doi.org/10.1002/met

Yang, C., Chandler, R. E., Isham, V. S., \& Wheater, H. S. (2005). Spatial-temporal rainfall simulation using generalized linear models. Water Resources Research, 41(11), 113. http://doi.org/10.1029/2004WR003739 